Revista de Psicología de la PUCP. Vol. XVIIl, 2, 2000

\title{
Personalidad, ansiedad estado-rasgo e ingreso a la universidad en alumnos preuniversitarios
}

\author{
Isabel Niño de Guzmán², Arturo Calderón, Marisa Escalante, \\ Tula Lira, Roxanna Morote, Lourdes Ruda ${ }^{3}$ \\ Pontificia Universidad Católica del Perú
}

\begin{abstract}
Estudio correlacional que identifica las dimensiones de personalidad, el tipo de ansiedad y las características sociodemográficas de alumnos de un centro preuniversitario. Se trabajó con 318 participantes de ambos sexos ( $43.8 \%$ mujeres y $56.2 \%$ hombres), que entre 16 y 19 años. Se los evaluó con los siguientes instrumentos: (a) NEO PI-R de Costa y McCrae (1992), (b) Inventario de Ansiedad Estado-Rasgo de Spielberger (IDARE, 1975) y (c) reporte de los tutores. Los resultados confirman la presencia de una estructura factorial básica de cinco dimensiones. Asimismo, revelan correlaciones significativas entre el C.I., caracteristicas de personalidad asociadas al factor conciencia e ingreso a la universidad. Entre las facetas que correlacionan con el ingreso destaca la autodisciplina. Finalmente, se discuten los resultados.
\end{abstract}

Palabras claves: personalidad, ansiedad estado y ansiedad rasgo, ingreso a la universidad.

\section{Personality, state-trait anxiety and admission to university in preuniversity students}

This correlational study identifies personality dimensions, type of anxiety and sociodemographic characteristics of students from a preuniversity center. It was conducted with 318 participants of both sexes ( $43.8 \%$ female and $56.2 \%$ male) whose ages range from 15 to 19 years old. They were assessed with the following instruments: Costa and McCrae's Personality Inventory NEO PI-R (1992), Spielberger's State-Trait Anxiety Inventory (STAI, 1975) and tutors' reports. Results confirm the presence of a basic factor structure of five dimensions: Likewise, they also reveal significant correlations among Intellectual Quotient (IQ), personality characteristics related to Conscientiousness factor and admission to university. Self-discipline stands out among the facets that correlate with admission. Finally, results are discussed.

Key words: personality, state and trait anxiety, and admission to university.

1. Se agradece a los alumnos de la Especialidad Clínica que colaboraron con la investigación.

2. Profesora Auxiliar. Magister en Psicología, especialista en evaluación y uso de pruebas psicológicas. Correo electrónico: inino@pucp.edu.pe.

3. Docentes de la sección de psicología. 

Estudios realizados en el medio han demostrado la relación entre los factores de personalidad y el rendimiento académico (Anchante, 1993; Claux, 1999). La habilidad mental o intelectual no resulta el único aspecto que determina un adecuado rendimiento; factores emocionales pueden incidir negativamente sobre éste. Por el contrario, características personales como interés por el logro, cumplimiento, competencia, orden, sentido del deber, apertura al conocimiento y autodisciplina influyen favorablemente sobre el rendimiento y la adaptación general.

Por otra parte, la asociación entre ansiedad y el rendimiento académico ha sido ampliamente documentada (Anchante, 1993). Un rendimiento deficiente puede traer como consecuencia estados emocionales como la disminución de la autoestima, anticipación al castigo, excesiva preocupación por el desempeño y sentimientos de vergüenza o culpa. Del mismo modo, estados ansiógenos intensos pueden producir bloqueos intelectuales y dificultades significativas en el desempeño académico.

El ingreso a la universidad constituye una meta inmediata y prioritaria para el estudiante. La decisión de seguir una carrera profesional va acompañada por presiones internas propias del desarrollo y por presiones externas tales como las expectativas familiares. La aprobación del examen constituye quizás el factor ansiógeno de mayor intensidad, pero su impacto puede disminuir gracias a los recursos personales (características de personalidad, manejo de la ansiedad, recursos intelectuales) y al soporte social con que pueda contar el postulante.

La evaluación del estado emocional y personal de los estudiantes, asociada a la de los hábitos de estudio, habilidades cognitivas y rendimiento académico, permite un panorama del afronte de la situación. 
Esto resulta de mucha utilidad para el desarrollo de programas de prevención y soporte en una institución preuniversitaria. Una apreciación subjetiva del tutor acompañada de una evaluación objetiva con instrumentos psicológicos puede brindar información relevante para propósitos descriptivos, predictivos y preventivos.

Se plantea como primer objetivo del trabajo la descripción de las dimensiones de personalidad y el tipo de ansiedad predominante en los participantes; en segundo lugar, se trata de determinar las relaciones entre ambos aspectos. Finalmente se evalúa la asociación de variables sociodemográficas (sexo, edad, colegio de procedencia, facultad y número de veces que se postula, grupo, nivel intelectual, y modalidad de ingreso) con el ingreso a la universidad.

\section{Metodología}

Se trata de una investigación ex post facto, de tipo correlacional con elementos descriptivos (Hernández, Fernández y Baptista, 1994), con un diseño no experimental, transeccional.

\section{Participantes}

La muestra quedó conformada por 318 jóvenes ${ }^{4}$, no escolares y escolares de quinto de media, de ambos sexos, 16 años como mínimo, postulantes a programas de Letras y Ciencias, procedentes de colegio de tipo laico, religioso, nacional y particular. Se utilizó un muestreo de tipo probabilístico no intencional, en función de los objetivos del estudio.

4. Se descartaron aquellos casos que no respondieron o que respondieron mal. 
Personalidad, ansiedad estado-rasgo e ingreso a la universidad...

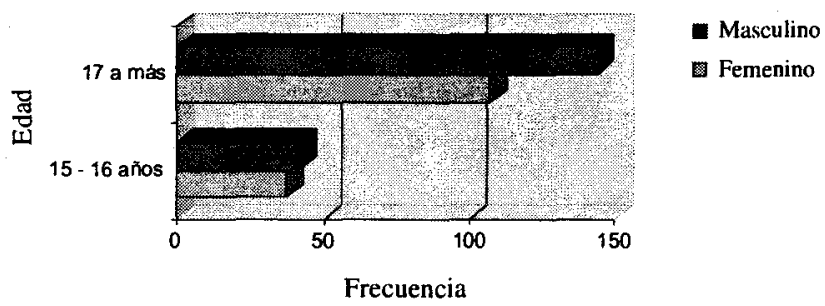

Figura 1. Distribución de la muestra por edad y sexo.

El grupo resulta altamente representativo del universo de referencia y tal como se puede apreciar en la Figura 1 se encuentra principalmente conformado por jóvenes de 17 años o más, existiendo un predominio de hombres con respecto al de mujeres. En la Figura 2 se observa que el $70 \%$ son no escolares, y el $30 \%$ pertenecen al grupo de escolares.

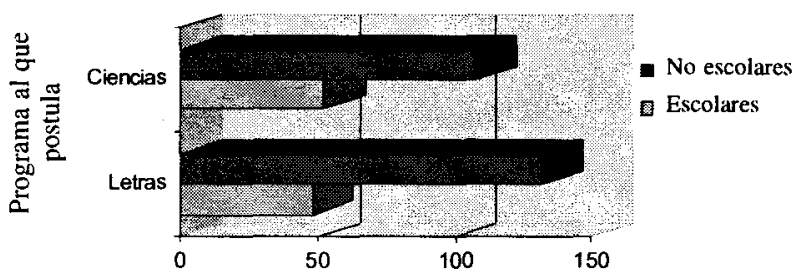

Frecuencia

Figura 2. Distribución de la muestra según ciclo y programa al que postula.

Casi la mitad del grupo (49\%) postula por primera vez; un 32 $\%$ lo intenta por segunda, y el $19 \%$ restante ha rendido el examen en más de dos ocasiones, como se observa en el Cuadro 1. La mayoría proviene de Lima y/o ha vivido por lo menos cinco años en ella, tal como aparece en el Cuadro 2. 
Isabel Niño de Guzmán et al.

\section{Cuadro 1}

Participantes según colegio de procedencia y número de veces que postulan $(N=3 / 8)$

\begin{tabular}{|lrrrrrrrrr|}
\hline & \multicolumn{9}{c}{ Veces que postula } \\
Colegio procedencia & \multicolumn{1}{c}{ Una vez } & \multicolumn{2}{c|}{ Dos veces } & \multicolumn{2}{c|}{ Más de dos } & \multicolumn{2}{c|}{ Total } \\
& $f$ & $\%$ & \multicolumn{1}{c}{$f$} & $\%$ & \multicolumn{1}{c|}{$f$} & $\%$ & \multicolumn{1}{c|}{$f$} & $\%$ \\
\hline Estatal & 22 & 14.3 & 12 & 12.0 & 6 & 9.5 & 40 & 12.6 \\
Privado & 70 & 45.5 & 51 & 51.0 & 33 & 52.4 & 154 & 48.6 \\
Privado religioso & 62 & 40.3 & 37 & 37.0 & 24 & 38.1 & 123 & 39.8 \\
Total & 154 & 100.0 & 100 & 100.0 & 63 & 100.0 & 317 & 100.0 \\
\hline
\end{tabular}

Casos perdidos $=1$

\section{Cuadro 2}

Participantes según lugar de nacimiento y tiempo de residencia en Lima $(N=318)$

\begin{tabular}{|c|c|c|c|c|c|c|c|c|}
\hline \multirow{2}{*}{\multicolumn{2}{|c|}{$\begin{array}{l}\text { Tiempo de residencia } \\
\text { en Lima }\end{array}$}} & Lima & \multicolumn{2}{|c|}{ Provincias } & \multicolumn{2}{|c|}{ Extranjero } & \multicolumn{2}{|c|}{ Total } \\
\hline & & $\%$ & $f$ & $\%$ & $f$ & $\%$ & $f$ & $\%$ \\
\hline - 2 años & 10 & 4.1 & 33 & 62.3 & 1 & 16.7 & 44 & 14.7 \\
\hline 3-4 aก̃os & 1 & 0.4 & 5 & 9.4 & 1 & 16.7 & 7 & 2.3 \\
\hline 5 o más años & 230 & 95.5 & 15 & 28.3 & 4 & 66.7 & 249 & 83.0 \\
\hline Total & 258 & 100.0 & 60 & 100.0 & 6 & 100.0 & 300 & 100.0 \\
\hline
\end{tabular}

Casos perdidos $=18$.

Se puede apreciar en la Figura 3 que el $56 \%$ obtuvo un resultado favorable en el ingreso. Un porcentaje menor no ingresó (30\%) o no se presentó al examen (14\%).

En cuanto al CI, la mayor parte del grupo se encuentra dentro de las categorías promedio y promedio superior. No se encuentran diferencias significativas entre hombres y mujeres. 
Personalidad, ansiedad estado-rasgo e ingreso a la universidad...

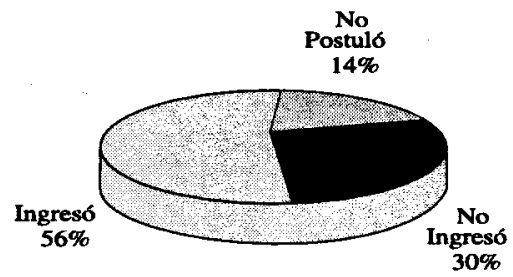

Figura 3. Distribución de la muestra según ingreso.

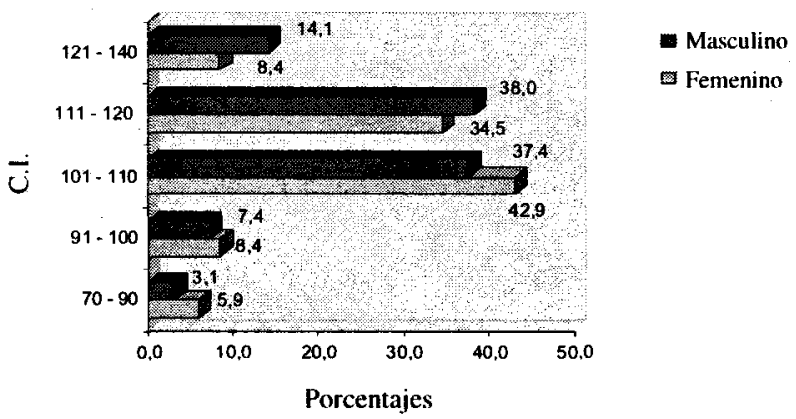

Figura 4. Distribución de la muestra según coeficiente intelectual y sexo.

\section{Instrumentos}

1. El Inventario de los Cinco Factores de Personalidad NEO PI-R (forma S) de Costa y Mc Crae (1992), adaptado por Cassaretto en 1999 para estudiantes universitarios de la PUCP, evalúa las cinco mayores dimensiones de la personalidad normal. La forma autoadministrada (S) consta de 240 ítems que se responden en una escala de cinco puntos. Costa y Mc Crae (1992) reportan un coeficiente alfa de confiabilidad de 0.56 a 0.81 en las escalas, así como la validez convergente, discriminante y de criterio externo del instrumento. El test distingue seis facetas y treinta rasgos de personalidad, los cuales se definen del siguiente modo: 
Extraversión: calidez (E1), gregarismo (E2), asertividad (E3), actividad (E4), búsqueda de sensaciones (E5), emociones positivas (E6). Agradabilidad: confianza (A1), franqueza (A2), altruismo (A3), complacencia (A4), modestia (A5), benevolencia (A6).

Conciencia: competencia $(\mathrm{C} 1)$, orden (C2), sentido del deber (C3), aspiraciones de logro (C4), autodisciplina (C5), reflexión (C6).

Neuroticismo: ansiedad (N1), hostilidad colérica (N2), depresión (N3), ansiedad social (N4), impulsividad (N5), vulnerabilidad (N6). Apertura: fantasía (O1), estética $(\mathrm{O} 2)$, sentimientos $(\mathrm{O} 3)$, acciones $(\mathrm{O} 4)$, ideas $(\mathrm{O5})$, valores $(\mathrm{O6})$.

2. El Inventario de Autoevaluación Rasgo-Estado IDARE, de Spielberger y Díaz Guerrero, construido para la medición de la ansiedad en población normal y clínica, consta de dos escalas de autoevaluación que miden dos dimensiones de la ansiedad: AnsiedadRasgo (SXR) y Ansiedad- Estado (SXE). La primera ha sido utilizada para la identificación de estudiantes de bachillerato y universitarios propensos a la ansiedad, y para evaluar el nivel de ansiedad en estudiantes que requieren servicios de consejo u orientación psicológica. La segunda indica el nivel de malestar transitorio que se expresa a través de sentimientos de tensión y aprensión subjetivamente percibidos, y de un aumento de la actividad del sistema nervioso autónomo (Spielberger y Díaz-Guerrero, 1975). Se ha determinado la validez concurrente de la prueba mediante otros inventarios de ansiedad Se reporta una confiabilidad original de 0.83 a 0.92 para la escala SXE y puntajes igualmente altos para la escala SXR. En el Perú la confiabilidad fue trabajada para universitarios por Anchante en 1993, Rojas en 1997, Chau en 1998 y Avendaño en 1999.

3. La evaluación del tutor constaba de una plantilla para la descripción objetiva y cualitativa de rasgos de personalidad y tipo de ansiedad predominante con un listado de descriptores de los alumnos según el criterio de cada tutor.

4. La ficha demográfica, con la cual se recogieron datos demográficos del grupo tales como edad, sexo, lugar de nacimiento, tiempo de permanencia en Lima, facultad a la que postula el 
Personalidad, ansiedad estado-rasgo e ingreso a la universidad...

alumno, ciclo, tipo de colegio, número de veces que se postula, modalidad de ingreso. Incluye la estimación del nivel intelectual de acuerdo al test de Madurez Mental de California (previamente evaluado por el Departamento Psicopedagógico del Centro).

\section{Procedimiento}

La investigación se llevó a cabo en dos etapas. En la primera, luego de la preparación de los evaluadores, se estableció contacto con los participantes. Se explicó el propósito de la investigación, se solicitó el consentimiento, se garantizó la confidencialidad y se aplicó las pruebas colectivamente. En la segunda etapa se capacitó a los tutores para la calificación de sus alumnos a través de la plantilla de corrección, se registraron los resultados de admisión y el CI y se procesaron estadísticamente los datos.

\section{Resultados}

Inicialmente se obtuvo una composición factorial de seis factores. El sexto, conformado por algunas facetas de apertura $(\mathrm{O} 4, \mathrm{O6})$ y de agradabilidad (A6), revela una correlación significativa con otros factores, sobre todo con el quinto. Por ello se llevó a cabo un nuevo análisis que consideró sólo cinco factores con valores eigen mayores a 2.31, tal como se aprecia en el Cuadro 3.

\section{Cuadro 3}

Análisis factorial del NEO PI- R: Valores eigen y porcentajes de varianza explicada $(N=318)$

\begin{tabular}{|cccc|}
\hline Factores & Valor Eigen & \% de varianza explicada & $\%$ de varianza explicada acumulada \\
\hline 1 & 4.16 & 13.86 & 13.86 \\
2 & 3.84 & 12.80 & 26.66 \\
3 & 3.66 & 12.22 & 38.88 \\
4 & 3.32 & 11.08 & 49.96 \\
5 & 2.31 & 7.72 & 57.67 \\
\hline
\end{tabular}


Todos los factores excepto Apertura $(\mathrm{O})$, mantienen la composición factorial y las facetas según lo señalado en diversos estudios norteamericanos (Costa y McCrae, en Ter Laak, 1996). En el Cuadro 4 se aprecia la matriz factorial en mayor detalle. El primer factor se compone por las facetas de la dimensión Conciencia y se adhiere vulnerabilidad (N6), esta última con una mayor carga en el tercer factor. En el segundo, tercer y cuarto factor se organizaron todas las facetas de las dimensiones $E x$ traversión, Neuroticismo y Agradabilidad.

Al cuarto factor se suman las facetas gregarismo (E1) y ansiedad colérica (N2). Sentimientos (O3) presenta una mayor carga en el quinto factor, y se mantiene en este. Calidez (E1) y ansiedad colérica (N2) permanecen en segundo y tercero respectivamente. En el quinto se agrupan cinco facetas de Apertura; acciones (O4) es la única no significativa. El primer factor corresponde a Conciencia, el segundo a Extraversión, el tercero a Neuroticismo, el cuarto a Agradabilidad, y el quinto a Apertura. Los puntajes más elevados aparecen en el segundo y el tercero y en las facetas altruismo (A3), calidez (E1), sentido del deber (C3) y aspiración de logro (C4). Los más bajos, al tercero y a las facetas hostilidad colérica (N2), apertura a acciones ( $\mathrm{O} 4)$, ansiedad social (N4), depresión (N3) y complacencia (A4).

En el Cuadro 5 aparece la conformación de las escalas y facetas. Extraversión obtiene la media más alta $(M=115.6)$ seguida por el factor Conciencia $(M=112.7)$.

En todos los factores se observan diferencias significativas asociadas a la variable sexo, como lo muestra el Cuadro 6. En el caso de las facetas las diferencias son significativas para A1, N6, E1, E2, E6, $\mathrm{O} 2, \mathrm{O} 3, \mathrm{~A} 2, \mathrm{~A} 3, \mathrm{~A} 5, \mathrm{~A} 6, \mathrm{C} 1, \mathrm{C} 3, \mathrm{C} 4$ y $\mathrm{C}^{5}$. Los puntajes de las mujeres son superiores que los de los hombres.

5. Confianza, vulnerabilidad, calidez, gregarismo, emociones, estética, sentimientos, franqueza, altruismo, modestia, benevolencia, competencia, s. del deber, a. de logro y autodisciplina 
Personalidad, ansiedad estado-rasgo e ingreso a la universidad...

\section{Cuadro 4}

Composición factorial del NEOPI-R a partir de la rotación varimax: cinco factores*

\begin{tabular}{|c|c|c|c|c|c|}
\hline \multirow[b]{2}{*}{ Facetas } & \multicolumn{4}{|c|}{$\begin{array}{l}\text { Factores } \\
N=318\end{array}$} & \multirow[b]{2}{*}{ v } \\
\hline & I & II & III & IV & \\
\hline & Conciencia & Extroversión & Neuroticismo & Agradabilidad & Apertura \\
\hline FC4 & 0.81 & 0.18 & -0.08 & -0.01 & 0.05 \\
\hline FC5 & 0.77 & -0.01 & -0.32 & 0.03 & -0.02 \\
\hline $\mathrm{FC} 3$ & 0.77 & -0.05 & -0.01 & 0.26 & -0.07 \\
\hline $\mathrm{FCl}$ & 0.71 & 0.20 & -0.28 & 0.14 & 0.10 \\
\hline $\mathrm{FC} 2$ & 0.68 & 0.03 & -0.05 & -0.12 & -0.18 \\
\hline FC6 & 0.58 & -0.25 & -0.33 & 0.27 & -0.06 \\
\hline FE2 & -0.16 & 0.76 & -0.14 & 0.18 & -0.06 \\
\hline FE6 & 0.11 & 0.73 & -0.12 & 0.25 & 0.11 \\
\hline FE1 & 0.05 & 0.73 & -0.02 & 0.48 & 0.05 \\
\hline FE4 & 0.30 & 0.64 & -0.13 & -0.13 & 0.17 \\
\hline FE5 & -0.12 & 0.57 & -0.07 & -0.19 & 0.16 \\
\hline FE3 & 0.37 & 0.55 & -0.14 & -0.25 & 0.24 \\
\hline FNI & -0.06 & -0.02 & 0.82 & 0.01 & 0.09 \\
\hline FN3 & -0.28 & -0.24 & 0.76 & -0.07 & 0.12 \\
\hline FN6 & -0.41 & -0.08 & 0.71 & -0.05 & -0.08 \\
\hline FN2 & -0.07 & -0.18 & 0.67 & -0.46 & 0.00 \\
\hline FN4 & -0.16 & -0.39 & 0.66 & 0.08 & -0.10 \\
\hline FN5 & -0.24 & 0.32 & 0.52 & -0.29 & 0.23 \\
\hline FA4 & 0.10 & -0.13 & -0.26 & 0.70 & 0.04 \\
\hline FA3 & 0.14 & 0.35 & 0.00 & 0.69 & 0.10 \\
\hline FA2 & 0.06 & -0.20 & -0.05 & 0.69 & -0.04 \\
\hline FA1 & -0.02 & 0.27 & -0.17 & 0.63 & -0.01 \\
\hline FA6 & 0.22 & 0.26 & 0.25 & 0.52 & 0.04 \\
\hline FA5 & -0.28 & -0.31 & 0.06 & 0.45 & -0.02 \\
\hline $\mathrm{FO} 2$ & 0.08 & 0.00 & 0.18 & 0.24 & 0.75 \\
\hline FO5 & 0.34 & -0.08 & -0.06 & 0.06 & 0.71 \\
\hline $\mathrm{FO} 3$ & 0.06 & 0.41 & 0.29 & -0.03 & 0.58 \\
\hline $\mathrm{FO} 1$ & -0.24 & 0.19 & 0.15 & -0.01 & 0.52 \\
\hline FO6 & -0.23 & 0.07 & -0.12 & -0.08 & 0.45 \\
\hline FO4 & -0.14 & 0.22 & -0.26 & -0.11 & 0.38 \\
\hline
\end{tabular}

Nota. * Se consideran significativas las facetas con cargas factoriales superiores a \pm .40 . 


\section{Cuadro 5}

Distribución de escalas y facetas del NEOPI-R: media y desviación estándar

\begin{tabular}{|lcc|}
\hline \multicolumn{1}{c}{ Escalas del NEO PI-R } & $M$ & $D E$ \\
\cline { 2 - 3 } & $N=318$ & \\
\hline N : Neuroticismo & 92.7 & 20.6 \\
E : Extroversión & 115.6 & 18.8 \\
O : Apertura & 108.9 & 15.1 \\
A : Agradabilidad & 106.6 & 15.4 \\
C : Conciencia & 112.7 & 19.3 \\
\hline FN1 : Ansiedad & 17.3 & 4.5 \\
FN2 : Ansiedad colérica & 14.5 & 4.7 \\
FN3 : Depresión & 15.3 & 5.2 \\
FN4 : Ansiedad social & 15.0 & 4.5 \\
FN5 : Impulsividad & 17.1 & 4.1 \\
FN6 : Vulnerabilidad & 13.5 & 4.3 \\
\hline FE1 : Calidez & 21.2 & 4.7 \\
FE2 : Gregarismo & 18.9 & 4.9 \\
FE3 : Asertividad & 16.9 & 4.3 \\
FE4 : Actividad & 17.6 & 3.2 \\
FE5 : Búsqueda de sensaciones & 20.2 & 4.3 \\
FE6 : Emociones positivas & 20.6 & 5.1 \\
\hline FO1 : Fantasía & 19.3 & 4.3 \\
FO2 : Estética & 18.8 & 5.3 \\
FO3 : Sentimientos & 19.3 & 3.9 \\
FO4 : Acciones & 14.9 & 2.7 \\
FO5 : Ideas & 18.6 & 5.3 \\
FO6 : Valores & 18.0 & 2.7 \\
\hline FA1 : Confianza & 16.1 & 4.2 \\
FA2 : Franqueza & 17.2 & 4.6 \\
FA3 : Altruismo & 21.8 & 3.8 \\
FA4 : Complacencia & 15.4 & 4.4 \\
FA5 : Modestia & 15.9 & 4.0 \\
FA6 : Benevolencia & 20.1 & 2.7 \\
\hline FC1 : Competencia & 19.9 & 3.7 \\
FC2 : Orden & 16.7 & 3.8 \\
FC3 : Sentido del deber & 21.1 & 4.1 \\
FC4 : Aspiraciones de logro & 21.0 & 4.4 \\
FC5 : Autodisciplina & 16.6 & 4.7 \\
FC6 : Reflexión & 17.3 & 4.6 \\
\hline
\end{tabular}


Personalidad, ansiedad estado-rasgo e ingreso a la universidad...

\section{Cuadro 6}

Diferencias según sexo

\begin{tabular}{|c|c|c|c|c|c|c|}
\hline \multirow[t]{2}{*}{ Dimensiones y facetas ${ }^{*}$} & \multicolumn{2}{|c|}{$\begin{array}{c}\text { Femenino } \\
n=137\end{array}$} & \multicolumn{2}{|c|}{$\begin{array}{c}\text { Masculino } \\
n=180\end{array}$} & \multirow[b]{2}{*}{$Z$} & \multirow[b]{2}{*}{$d$} \\
\hline & $M$ & $D E$ & $M$ & $D E$ & & \\
\hline $\mathrm{N}:$ Neuroticismo & 95.9 & 23.0 & 90.2 & 18.1 & 2.39 & 0.28 \\
\hline E : Extroversión & 119.1 & 19.4 & 112.9 & 18.0 & 2.91 & 0.33 \\
\hline$O:$ Apertura & 111.1 & 15.3 & 107.1 & 14.7 & 2.32 & 0.26 \\
\hline A : Agradabilidad & 110.5 & 15.9 & 103.8 & 14.4 & 3.88 & 0.45 \\
\hline $\mathrm{C}:$ Conciencia & 116.5 & 20.2 & 110.0 & 18.0 & 2.96 & 0.34 \\
\hline FN1 : Ansiedad & 18.5 & 4.5 & 16.5 & 4.3 & 3.94 & 0.45 \\
\hline FN2 : Ansiedad colérica & 14.8 & 5.1 & 14.2 & 4.3 & 1.08 & 0.13 \\
\hline FN3 : Depresión & 15.7 & 5.7 & 15.0 & 4.8 & 1.18 & 0.14 \\
\hline FN4 : Ansiedad social & 15.5 & 4.7 & 14.6 & 4.3 & 1.72 & 0.20 \\
\hline FN5 : Impulsividad & 17.1 & 4.7 & 17.0 & 3.6 & 0.17 & 0.02 \\
\hline FN6: Vulnerabilidad & 14.4 & 4.6 & 12.9 & 4.0 & 2.98 & 0.34 \\
\hline FE1 : Calidez & 22.3 & 4.5 & 20.4 & 4.6 & 3.67 & 0.41 \\
\hline FE2 : Gregarismo & 19.6 & 5.2 & 18.4 & 4.6 & 2.20 & 0.25 \\
\hline FE3 : Asertividad & 17.4 & 4.6 & 16.6 & 4.0 & 1.76 & 0.20 \\
\hline FE4 : Actividad & 17.9 & 3.5 & 17.3 & 2.9 & 1.62 & 0.19 \\
\hline FE5: Búsqueda de sensaciones & es 19.8 & 4.6 & 20.6 & 4.0 & -1.70 & 0.20 \\
\hline FE6 : Emociones positivas & 22.0 & 5.0 & 19.6 & 5.0 & 4.33 & 0.49 \\
\hline FO1 :Fantasía & 19.0 & 4.7 & 19.4 & 4.0 & -0.87 & 0.10 \\
\hline FO2 :Estética & 20.5 & 5.0 & 17.5 & 5.2 & 5.20 & 0.59 \\
\hline FO3 :Sentimientos & 20.0 & 4.1 & 18.8 & 3.8 & 2.74 & 0.31 \\
\hline FO4 : Acciones & 14.7 & 2.7 & 15.0 & 2.7 & -0.99 & 0.11 \\
\hline FO5 : Ideas & 18.6 & 5.6 & 18.7 & 5.2 & -0.08 & 0.01 \\
\hline FO6 : Valores & 18.3 & 3.0 & 17.8 & 2.5 & 1.63 & 0.19 \\
\hline FAl : Confianza & 16.3 & 4.2 & 16.1 & 4.2 & 0.40 & 0.05 \\
\hline FA2 : Franqueza & 18.4 & 4.7 & 16.3 & 4.3 & 4. 10 & 0.47 \\
\hline FA3 : Altruismo & 22.6 & 4. 1 & 21.2 & 3.6 & 3.33 & 0.38 \\
\hline FA4 : Complacencia & 15.9 & 4.6 & 15.1 & 4. 1 & 1.76 & 0.20 \\
\hline FA5 : Modestia & 16.5 & 4.0 & 15.5 & 4.1 & 2.27 & 0.26 \\
\hline FA6: Benevolencia & 20.7 & 2.5 & 19.7 & 2.8 & 3.44 & 0.38 \\
\hline FC1 : Competencia & 20.5 & 3.8 & 19.6 & 3.5 & 2.13 & 0.24 \\
\hline FC2 : Orden & 17.2 & 3.9 & 16.4 & 3.6 & 1.87 & 0.21 \\
\hline FC3 : Sentido del deber & 22.0 & 4.2 & 20.4 & 3.9 & 3.51 & 0.40 \\
\hline FC4 : Aspiraciones de logro & 21.8 & 4.6 & 20.4 & 4. 2 & 2.69 & 0.31 \\
\hline FC5 : Autodisciplina & 17.4 & 4.9 & 16.1 & 4.4 & 2.29 & 0.26 \\
\hline FC6 : Reflexión & 17.6 & 4.9 & 17.1 & 4.3 & 1.11 & 0.13 \\
\hline
\end{tabular}

Nota. *Significativo: $z> \pm 1.96$ y $d>0.25$ (diferencia pequeña). Casos perdidos $=1$ 
Cuadro 7

Diferencias según lugar de nacimiento, programa y ciclo

\begin{tabular}{|c|c|c|c|c|c|c|}
\hline \multirow[t]{2}{*}{ Escala } & \multicolumn{2}{|c|}{$\begin{array}{l}\text { Lugar de nacimiento } \\
\text { Lima: } n=251 \\
\text { Provincia: } n=56\end{array}$} & \multicolumn{2}{|c|}{$\begin{array}{c}\text { Programa } \\
\text { Letras: } n=167 \\
\text { Ciencias: } n=146\end{array}$} & \multicolumn{2}{|c|}{$\begin{array}{c}\text { Ciclo } \\
\text { Escolares: } n=95 \\
\text { No escolares: } n=223\end{array}$} \\
\hline & $Z$ & $d$ & $Z$ & $d$ & $Z$ & $d$ \\
\hline $\mathbf{N}$ & & & & & & \\
\hline $\mathbf{E}$ & 2.31 & 0.34 & & & & \\
\hline $\mathrm{O}$ & & & 5.08 & 0.57 & & \\
\hline A & & & & & & \\
\hline C & & & & & & \\
\hline FN2 & & & & & -1.98 & 0.24 \\
\hline FN4 & & & & & -2.2 & 0.27 \\
\hline FE2 & 3.09 & 0.47 & & & & \\
\hline FE4 & 2.64 & 0.38 & & & 2.48 & 0.31 \\
\hline FO1 & & & 2.83 & 0.32 & & \\
\hline $\mathrm{FO} 2$ & & & 6.26 & 0.71 & & \\
\hline FO3 & & & 2.89 & 0.32 & & \\
\hline FO5 & & & 2.53 & 0.29 & & \\
\hline FO6 & & & 3.38 & 0.38 & & \\
\hline FA4 & -2.93 & 0.38 & & & & \\
\hline $\mathrm{FC} 2$ & -2.07 & 0.32 & & & & \\
\hline FC5 & & & & & 2. 10 & 0.27 \\
\hline
\end{tabular}

Nota. Significativo: $z> \pm 1.96$ y $d>.25$ (diferencia pequeña). Casos perdidos $=16$.

Las variables lugar de nacimiento y programa marcan diferencias en Extraversión y Apertura respectivamente (Cuadro 7). Existen diferencias entre los alumnos nacidos en Lima y en provincia en las facetas $\mathrm{E} 2, \mathrm{E} 4, \mathrm{~A} 4$ y $\mathrm{C2}^{6}$. El programa al que postulan afecta a cinco de las seis facetas de Apertura: $\mathrm{O} 1, \mathrm{O} 2, \mathrm{O} 3, \mathrm{O} 5$ y $\mathrm{O}^{7}$. El ciclo se asocia a diferencias entre escolares y no escolares en N2, N4, E4 y $\mathrm{C5}^{8}$.

6. Gregarismo, actividad, complacencia, orden.

7. Fantasía, estética, sentimientos, ideas, valores

8. A. colérica, ansiedad, social, actividad, autodisciplina. 
Personalidad, ansiedad estado-rasgo e ingreso a la universidad...

Entre los colegios estatales y privados se evidencian diferencias significativas en el factor Conciencia y específicamente en las facetas competencia (C1), sentido del deber (C3) y reflexión (C6). También existen diferencias significativas en las facetas complacencia (A4), fantasía (01), sentimientos (O3) y acciones (O4). La comparación entre los colegios estatales y privados religiosos revela diferencias en la dimensión Extraversión así como en las facetas gregarismo (E2), complacencia (A4), fantasía (O1), y acciones (04). Las diferencias en colegios privados únicamente resultan significativas en la faceta calidez (E1).

\section{Cuadro 8}

Diferencias según colegio de procedencia

\begin{tabular}{|c|c|c|c|c|c|c|}
\hline \multirow[t]{3}{*}{ Escala } & \multicolumn{6}{|c|}{ Colegio de procedencia } \\
\hline & \multicolumn{2}{|c|}{$\begin{array}{c}\text { Estatal: } n=40 ; \\
\text { Estatal - Privado }\end{array}$} & \multicolumn{2}{|c|}{$\begin{array}{c}\text { Privado: } n=154 \\
\text { Estatal - P, Religioso }\end{array}$} & \multicolumn{2}{|c|}{$\begin{array}{l}\text { P. Religioso: } n=123 \\
\text { Privado - P, Religioso }\end{array}$} \\
\hline & $Z$ & $d$ & $Z$ & $d$ & $Z$ & $d$ \\
\hline \multicolumn{7}{|c|}{ Neuroticismo } \\
\hline Extroversión & & & -2.0 & 0.35 & & \\
\hline \multicolumn{7}{|l|}{ Apertura } \\
\hline \multicolumn{7}{|c|}{ Agradabilidad } \\
\hline Conciencia & 2.33 & 0.39 & & & & \\
\hline FE1 & & & & & -2.04 & 0.25 \\
\hline FE2 & & & -2.36 & 0.44 & & \\
\hline FO1 & -2.24 & 0.34 & -2.38 & 0.37 & & \\
\hline FO3 & -1.97 & 0.33 & & & & \\
\hline FO4 & -2.21 & 0.38 & -2.04 & 0.36 & & \\
\hline FA4 & 2.32 & 0.39 & 2.4 & 0.39 & & \\
\hline FC1 & 2.03 & 0.34 & & & & \\
\hline FC3 & 2.60 & 0.47 & & & & \\
\hline FC6 & 2.3 & 0.4 & & & & \\
\hline
\end{tabular}

Nota. Significativo: $z> \pm 1.96$ y $d>.25$ (diferencia pequeña). Casos perdidos $=1$. 
En cuanto a los puntajes del IDARE, ambas escalas se encuentran dentro del promedio esperable (Anchante, 1993), entre el percentil 45.8 y 41.5 respectivamente.

\section{Cuadro 9}

Distribución del IDARE: media y desviación estándar

\begin{tabular}{|lcc|}
\hline Escalas del IDARE & $M$ & $D E$ \\
& \multicolumn{2}{c|}{$N=314$} \\
\hline Ansiedad Rasgo & 44.4 & 9.4 \\
Ansiedad Estado & 42.0 & 8.7 \\
\hline
\end{tabular}

Nota. Casos perdidos $=4$.

No hay diferencias significativas en los puntajes del IDARE según la edad, lugar de nacimiento y colegio de procedencia, pero sí aparecen diferencias significativas en ambas escalas en las variables sexo y ciclo. Las mujeres y los no escolares obtienen puntajes mayores tanto en la escala de Ansiedad-Rasgo como en la de Ansiedad-Estado. Los alumnos del programa de Letras presentan puntajes significativamente más altos únicamente en la escala de Ansiedad Estado, como aparece en el Cuadro 10.

\section{Cuadro 10}

Diferencias según sexo, programa y ciclo

\begin{tabular}{|lcccccc|}
\hline Escala & \multicolumn{2}{c}{ Sexo } & \multicolumn{2}{c|}{ Programa } & \multicolumn{2}{c|}{ Ciclo } \\
& $\begin{array}{c}\text { Hombres: } n=179 \\
\text { Mujeres: } n=134\end{array}$ & $\begin{array}{c}\text { Letras: } n=163 \\
\text { Ciencias: } n=143\end{array}$ & $\begin{array}{c}\text { Escolares: } n=91 \\
\text { No escolares: } n=223\end{array}$ \\
\cline { 2 - 8 } & $z$ & $d$ & $z$ & $d$ & $z$ & $d$ \\
\hline Ansiedad Rasgo* & 2.58 & 0.30 & - & - & -3.54 & 0.43 \\
Ansiedad Estado* & 2.38 & 0.28 & 2.14 & 0.24 & -2.66 & 0.31 \\
\hline
\end{tabular}

Nota. *Significativo: $z> \pm 1.96$ y $d>.25$ (diferencia pequeña). Casos perdidos: sexo $=5$; programa $=12 ;$ ciclo $=4$. 
Personalidad, ansiedad estado-rasgo e ingreso a la universidad...

El Cuadro 11 muestra sólo las facetas en que correlacionan las características personales autoreportadas por los alumnos y las percibidas por sus tutores.

\section{Cuadro 11}

Correlación NEO PI-R- autoreporte de alumnos-reporte de tutores

\begin{tabular}{|lcr|}
\hline \multicolumn{1}{|c}{ Factores y facetas del NEO-PI } & Correlación & $N$ \\
\hline Neuroticismo & - & 275 \\
Extraversión & $0.29^{*}$ & 274 \\
Apertura & $0.26^{*}$ & 276 \\
Agradabilidad & $0.21^{*}$ & 276 \\
Conciencia & $0.31^{*}$ & 275 \\
N3 : Depresión & $0.17^{*}$ & 102 \\
N5 : Impulsividad & $0.24^{*}$ & 66 \\
E1 : Calidez & $0.32^{*}$ & 133 \\
E2 : Gregarismo & $0.18^{*}$ & 132 \\
E4 : Actividad & $0.26^{*}$ & 61 \\
E6 : Emociones positivas & $0.38^{*}$ & 55 \\
O2 : Estética & $0.36^{*}$ & 47 \\
O6 : Valores & $0.24^{*}$ & 65 \\
A2 : Franqueza & $0.15^{*}$ & 155 \\
C1 : Competencia & $0.33^{*}$ & 75 \\
C3 : Sentido del deber & $0.33^{*}$ & 135 \\
C4 : Aspiración de logro & $0.33^{*}$ & 133 \\
C5 : Autodisciplina & $0.46^{*}$ & 65 \\
\hline
\end{tabular}

Nota. $p<.05$ (con un $95 \%$ de confianza).

Se analizó también las correlaciones entre factores y facetas del NEO PI- R y las facetas del IDARE. Se encuentran correlaciones significativas entre todos los factores del NEOPI- $R$ y ambas escalas del IDARE (Cuadro 12). No ocurre en el caso de las facetas E5, O4, 05, O6, A2, A5 y A6" y la Escala de Ansiedad- Rasgo. A su vez, las facetas E1, E5, O4, O5, O6, A2, A3, A5 y A6 ${ }^{10}$ no presentan una correlación significativa con la escala de Ansiedad- Estado.

9. Búsqueda de sensaciones, acciones, ideas, valores, franqueza, modestia, benevolencia.

10. Calidez, búsqueda de sensaciones, acciones, ideas, valores, franqueza, altruismo, modestia y benevolencia 


\section{Cuadro 12}

Correlación entre el NEOPI-R e IDARE.

\begin{tabular}{|lcc|}
\hline Factores y facetas NEO - PI- R & Ansiedad rasgo & Ansiedad estado \\
\cline { 2 - 3 } & \multicolumn{2}{c|}{$N=314$} \\
\hline Neuroticismo & 0.76 & 0.56 \\
Extraversión & -0.28 & -0.16 \\
Apertura & 0.15 & 0.13 \\
Agradabilidad & -0.19 & -0.12 \\
Conciencia & -0.39 & -0.34 \\
N1: Ansiedad & 0.64 & 0.47 \\
N2: Hostilidad colérica & 0.59 & 0.42 \\
N3: Depresión & 0.68 & 0.53 \\
N4: Ansiedad social & 0.5 & 0.31 \\
N5: Impulsividad & 0.38 & 0.32 \\
N6: Vulnerabilidad & 0.63 & 0.48 \\
E1: Calidez & -0.16 & - \\
E2: Gregarismo & -0.19 & -0.1 \\
E3: Asertividad & -0.23 & -0.14 \\
E4: Actividad & -0.26 & -0.13 \\
E5: Emociones & - & - \\
E6: Emociones positivas & -0.3 & -0.22 \\
O1: Fantasía & 0.1 & - \\
O2: Estética & 0.18 & 0.2 \\
O3: Sentimientos & 0.2 & 0.14 \\
O4: Acciones & - & - \\
O5: Ideas & - & - \\
O6: Valores & - & - \\
A1: Confianza & -0.25 & -0.17 \\
A2: Franqueza & - & - \\
A3: Altruismo & -0.17 & - \\
A4: Complacencia & -0.25 & -0.19 \\
A5: Modestia & - & - \\
A6: Benevolencia & - & - \\
C1: Competencia & -0.4 & -0.33 \\
C2: Orden & -0.2 & -0.17 \\
C3: Sentido del deber & -0.21 & -0.19 \\
C4: Aspiración de logro & -0.26 & -0.24 \\
C5: Autodisciplina & -0.41 & -0.35 \\
C6: Reflexión & -0.3 & -0.28 \\
\hline & & \\
Nota. $p<.05$ (con un & \\
\hline
\end{tabular}

Nota. $p<.05$ (con un $95 \%$ de confianza). Casos perdidos $=4$. 
Personalidad, ansiedad estado-rasgo e ingreso a la universidad...

La única correlación con nivel de significación $p<<.05$ aparece entre la Ansiedad- Estado autoreportada por los alumnos y la Ansiedad-Rasgo percibida por los tutores $(p=02)$. Los tutores estarían percibiendo la primera como un rasgo de los alumnos (Cuadro 13). La correlación, si bien significativa, señala una asociación débil entre ambas variables y resulta cuestionable pues podría ser por efecto del azar.

\section{Cuadro 13}

Correlación del IDARE con autoreporte de los alumnos y reporte de tutores

\begin{tabular}{|lcc|}
\hline IDARE Autoreporte & \multicolumn{2}{c|}{ IDARE según tutores $N=267$} \\
\cline { 2 - 3 } & Ansiedad rasgo & Ansiedad estado \\
\hline Ansiedad Rasgo & 0.09 & 0.00 \\
Ansiedad Estado & $0.12^{*}$ & 0.05 \\
\hline
\end{tabular}

Nota. $p<.05$ (con un $95 \%$ de confianza). Casos perdidos $=51$.

En el autoreporte de los alumnos Neuroticismo y Conciencia y las facetas $\mathrm{N} 3, \mathrm{~N} 6, \mathrm{O}, \mathrm{C3}, \mathrm{C} 4$ y $\mathrm{C}^{11}$ correlacionan significativamente con el ingreso. En el reporte de los tutores (Cuadro 14), la correlación con ingreso es significativa en Apertura, Agradabilidad y Conciencia y en N4, E3, E5, O5, A2, C1, C2, C3, $\mathrm{C} 4$ y $\mathrm{C}^{12}$.

11. Depresión, vulnerabilidad, ideas, sentido del deber, autodisciplina

12. Ansiedad social, asertividad, emociones, ideas, franqueza, competencia, orden, sentido del deber, a. de logro y autodisciplina 


\section{Cuadro 14}

Correlación entre autoreporte NEOPI - $R$ y reporte de los tutores con ingreso

\begin{tabular}{|lccr|}
\hline NEO-PI & Autoreporte-Ingreso & Tutores-Ingreso & $N$ \\
\hline Extroversión & - & - & - \\
Neuroticismo & -0.12 & - & - \\
Apertura & - & 0.24 & 269 \\
Agradabilidad & - & 0.18 & 269 \\
Conciencia & 0.14 & 0.55 & 268 \\
N3: Depresión & -0.16 & - & - \\
N4: Ansiedad social & - & 0.25 & 89 \\
N6: Vulnerabilidad & -0.15 & - & - \\
E3: Asertividad & - & 0.34 & 62 \\
E5: Emociones & - & -0.41 & 66 \\
O5: Ideas & 0.14 & 0.48 & 90 \\
A2: Franqueza & - & 0.19 & 149 \\
C1: Competencia & - & 0.51 & 74 \\
C2: Orden & - & 0.53 & 71 \\
C3: Sentido de deber & 0.17 & 0.48 & 130 \\
C4: Aspiración de logro & 0.16 & 0.70 & 132 \\
C5: Autodisciplina & 0.12 & 0.46 & 61 \\
\hline
\end{tabular}

Nota. $p<.05$ (con un $95 \%$ de confianza). Casos perdidos: autoreporte $=29$.

La única correlación significativa entre las variables del IDARE y el ingreso a la universidad se encuentra entre la Ansiedad - Estado autoreportada por alumnos e ingreso $(-0.12)$ que fue pequeña e inversamente proporcional. Mediante los análisis de correlación anteriores se identificaron una serie de asociaciones entre las variables de estudio y la variable ingreso. Dichas asociaciones pueden estar influenciadas por correlaciones al interior de las mismas variables. Para poder eliminar el efecto que una produce en la otra se procedió a realizar un análisis de regresión logística. Así, se puede observar la influencia de cada variable de manera independiente con el ingreso a la universidad.

El análisis fue realizado en cuatro etapas. Las variables autoreportadas por los alumnos y su coeficiente intelectual (CI) formaron 
Personalidad, ansiedad estado-rasgo e ingreso a la universidad...

parte del primer análisis. El resultado muestra una correlación significativa entre el factor Conciencia (facetas C3,C4 y C5), C.I. e ingreso, que muestran capacidad para clasificar correctamente el $67.3 \%$ de la muestra. El CI muestra una correlación parcial superior que Conciencia, revelando su mayor importancia dentro del análisis. La correlación con las variables Ansiedad Rasgo- y Estado, y los factores Neuroticismo, Extraversión, Apertura y Agradabilidad, mostró una asociación anulada por el efecto de las variables $\mathrm{C}$ y $\mathrm{CI}$.

En el segundo análisis se evaluó el efecto de las facetas y nuevamente aparece una faceta del factor Conciencia (C3) asociada al CI como una variable con poder explicativo. Ambas clasifican correctamente a un $71.54 \%$ de la muestra. Nuevamente el CI muestra una correlación parcial más fuerte, revelando una mayor importancia con respecto a la variable ingreso a la universidad. En el tercer análisis se evalúan las variables reportadas por los tutores. Una vez descartada su influencia mutua se encontró que nuevamente el factor $\mathrm{C}$ asociado al CI poseían valor explicativo, mostrando capacidad para clasificar correctamente a un $77 \%$ del grupo.

En cuanto a las variables demográficas se encontró una correlación alta entre el número de veces que el alumno postula y el ingreso, principalmente cuando se trataba de más de dos postulaciones. Sin embargo, al descartar el efecto de la variable CI, ésta perdía poder explicativo. El resultado final del análisis se presenta en el Cuadro 15 . Se concluye que las únicas variables que presentan una correlación positiva, significativa e independiente con el ingreso de los alumnos a la universidad son el factor $\mathrm{C}$ (Conciencia) reportado por los tutores y el $\mathrm{CI}$, que permiten determinar con un $78 \%$ de acierto el ingreso. 


\section{Cuadro 15}

Análisis de regresión logística entre las variables de investigación e ingreso a la universidad $(N=218)$

\begin{tabular}{|lccc|}
\hline Variables* & B & SE B & b $\beta$ \\
\hline Paso 1 & & & \\
$\quad$ Factor Conciencia reportado por el alumno (C) & 0.02 & 0.00 & $1.02^{*}$ \\
$\quad$ Coeficiente Intelectual (CI) & 0.11 & 0.01 & $1.11^{*}$ \\
Paso 2 & & & \\
$\quad$ Faceta autoreportada Sentido del deber (C3) & 0.11 & 0.03 & $1.12^{*}$ \\
$\quad$ Coeficiente intelectual (CI) & 0.11 & 0.01 & $1.11^{*}$ \\
Paso 3 & & & \\
$\quad$ Factor Conciencia reportado por el tutor (C) & 0.96 & 0.25 & $2.62^{*}$ \\
$\quad$ Coeficiente Intelectual CI & 0.08 & 0.02 & $1.08^{*}$ \\
Paso 4 & & & \\
$\quad$ Postula una vez & 0.29 & 0.27 & $1.34^{*}$ \\
$\quad$ Postula dos veces & 1.13 & 0.36 & $3.09^{*}$ \\
\hline
\end{tabular}

Nota. ${ }^{*} \mathrm{R}=0.11$ y 0.31 para el Paso $1 ; \mathrm{R}=0 ., 13$ y 0.31 para el Paso $2 ; \mathrm{R}=0.34$ y 0.20 para el Paso $3 ; R=0.00$ y $0.14(p<.05)$. Casos perdidos $=55$.

\section{Discusión}

Se ha verificado en diferentes investigaciones la existencia de las Cinco Grandes Dimensiones de la personalidad (John, 1990; Mc Crae y John, 1992). En nuestro estudio el análisis de los rasgos de personalidad según el NEO PI- R muestra que, tanto el número como la configuración factorial hallada en los participantes guarda coherencia con la originalmente propuesta por Costa y Mc Crae y aporta evidencia favorable con relación al modelo de los Cinco Factores.

Cabe señalar que si bien la composición de los factores coincide también con los hallazgos de Cassaretto en nuestro medio (1999), la estructura de las facetas no se reproduce con la misma exactitud. No se puede definir aún si este es un asunto de confiabilidad o se trata de diferencias de personalidad asociadas a un contexto cultural determinado. 
Personalidad, ansiedad estado-rasgo e ingreso a la universidad...

La más baja correlación se encuentra en la faceta de acciones, que podría sugerir cierta dificultad en el grupo para llevar a cabo actividades diferentes de las familiares y rutinarias, para animarse a aceptar lo nuevo. El resultado podría vincularse a la etapa de cambio e incertidumbre, que sin embargo no inhibe el interés por la afiliación y el trato social, ni por la búsqueda de reforzamiento en las interacciones sociales, como lo muestran los puntajes elevados en Extraversión.

Lo anterior se suma a los puntajes elevados en el factor Conciencia y las facetas de altruismo, calidez, sentido del deber y aspiraciones de logro. EI perfil corresponde al de personas enérgicas, caracterizadas por una conducta dirigida al manejo y organización del ambiente, a la solución de problemas mediante una actitud analítica y reflexiva y al completamiento de tareas (Watson y Lindgren, 1992). Indica el predominio de un estilo individual de trabajo y organización, con parámetros de diligencia y trabajo duro (Costa y McCrae, 1992) y formas de enfrentar las tareas de la vida orientadas a un alto nivel de logro académico (Digman y Takemoto-Chock,1981).

Conciencia es el factor con el que más se ha relacionado la motivación de logro, por su relación significativa con criterios formales de logro educacional y ocupacional. Las personas con alta Conciencia presentan elevadas metas personales debido a su convicción de obtenerlas (Cassareto, 1999), su tendencia a persistir en tareas que son un reto y en las que podría ser aplicado un patrón de excelencia. Esto es de esperar en un grupo de jóvenes normales, que han optado por presentarse a una institución conocida por el alto nivel de dificultad y exigencia que requiere para su ingreso, evaluados en una situación de vida que exige de ellos una alta competitividad. La preocupación por el bienestar común y los ideales del grupo aparece también alta, evidenciando que nos encontramos ante un grupo de jóvenes mental y emocionalmente saludables.

El factor Apertura, elevado en términos de algunas de sus facetas y asociado a variables específicas, hace referencia al pensamiento 
divergente; asimismo se considera un importante predictor del logro intelectual, la imaginación creativa, la adquisición del conocimiento y el desarrollo de intereses vocacionales (Costa y McCrae, 1992). Nuestro estudio da cuenta de un grupo interesado en formar parte de una institución formativa caracterizada por "la responsabilidad de formar integralmente como personas, es decir, como seres libres y pensantes, a los jóvenes que acuden a nuestro claustro" (Lerner, 2000, p, 63).

Por otra parte, los puntajes bajos en el factor Neuroticismo, y en las facetas de hostilidad colérica, apertura a las acciones, ansiedad social, depresión y complacencia, coinciden con los puntajes del inventario de Ansiedad-Estado y Ansiedad-Rasgo y reflejan a un grupo que, a pesar de estar sometido a una presión muy intensa, muestra un predominio de emociones positivas: calidez, gregarismo, asertividad, actividad, búsqueda de sensaciones, y amplitud de conciencia.

En las autodescripciones se encuentra una correlación positiva entre Ingreso y el factor Conciencia (sentido del deber, aspiración de logro, y autodisciplina), así como una correlación negativa con el factor Neuroticismo (depresión y vulnerabilidad). Este dato coincide con los hallazgos de Claux (1999), quien se refiere a la percepción de la personalidad como el atributo más relevante para predecir el logro académico en la escuela, asociado a factores mediadores como la imagen del yo ideal, el estatus sociométrico dentro de la clase (aceptación y rechazo de los pares), y otros factores externos al proceso como la calidad de enseñanza, materiales y métodos educacionales.

En cuanto al reporte de los tutores se observa que en sus términos el ingreso se asocia con los factores de Conciencia, Apertura y Agradabilidad. Esto se expresa también en las correlaciones positivas con las facetas asertividad, franqueza, competencia, orden, sentido del deber, aspiración de logro y autodisciplina. En cambio, las emociones correlacionan negativamente. Este resultado puede dar 
Personalidad, ansiedad estado-rasgo e ingreso a la universidad...

cuenta de la importancia de factores mediadores en el aprendizaje, uno de los cuales resulta la imagen que tiene el profesor del alumno.

Cabe mencionar que esta imagen del alumno percibida por el tutor no está desligada de la imagen que tiene el alumno de sí mismo. Definitivamente, el tutor identifica esta característica cuando forma parte de la autopercepción del alumno. Al parecer la influencia de esa percepción resulta un factor determinante en el Ingreso a la Universidad, por resultar altamente predictivo de la probabilidad de ingreso en el grupo evaluado.

En general, aún no se ha logrado determinar con precisión cuál de estas variables es la más importante o es el mejor predictor del logro académico. Por ello resulta importante deslindar el efecto específico de cada una con el ingreso y no únicamente las correlaciones de las variables entre sí. Se identificó una serie de asociaciones entre las variables de estudio y la variable ingreso, que podrían estar influenciadas por correlaciones al interior de las mismas variables. Los análisis de regresión logística y de correlación parcial permiten eliminar el efecto que una produce en la otra y observar la influencia de cada variable de manera independiente.

La correlación positiva hallada en el primer análisis entre el factor Conciencia, el coeficiente intelectual y el ingreso a la universidad era esperable, pero al eliminar el efecto de la variable C.I. sobre el factor Conciencia se encontró que esta variable no poseía valor explicativo significativo. Se concluye que las únicas variables que presentan correlación positiva, significativa e independiente con el ingreso de los alumnos a la universidad son el factor Conciencia reportado por los tutores y el C.I. en la medida que permiten determinar el ingreso de los alumnos con un $78 \%$ de acierto.

El tema de la validez convergente y discriminante de los constructos hace necesaria la utilización de procedimientos aplicables en contextos diferentes pero que además puedan recoger la particularidad 
de los contextos culturales (Ter Laak, 1996). Se observan diferencias significativas asociadas a la variable sexo en todos los factores, y en las facetas de vulnerabilidad, calidez, gregarismo, estética, emociones positivas, sentimientos, confianza, franqueza, altruismo, modestia, sentido del deber, aspiración de logro y autodisciplina. Estas no llegan a determinar diferencias en el ingreso, pero el hecho que todas reflejen puntajes superiores en las mujeres podría estar referido a rasgos comúnmente asociados al sexo femenino (sensibilidad por la belleza) y también a una percepción favorable de las jóvenes que se involucran en una situación de alta competitividad y exigencia, a su aspiración de logro.

Con respecto a la variable edad, debemos señalar que se reproduce la estructura factorial del modelo en un porcentaje de jóvenes de 15 y 16 años. Esta constituye la primera referencia en nuestro medio de las dimensiones en este grupo de edad a través del NEO PI$\mathrm{R}$, y coincide con hallazgos obtenidos a través de otros procedimientos como las descripciones Q-sort (Van Lieshout y Haselager, 1991) y listas de adjetivos (Goldberg, en Claux, 1999).

La variable lugar de nacimiento marca diferencias en Extraversión, y la variable programa en Apertura. Se encuentra diferencias entre los alumnos nacidos en Lima y en provincia en las facetas de gregarismo y actividad, así como complacencia y orden, que podrían expresar matices de la cultura. El programa al que postulan afecta a cinco de las seis facetas de la dimensión Apertura, lo que estaría reflejando asociaciones entre el campo de interés vocacional y personalidad, que coinciden con estudios hechos recientemente en nuestra población universitaria (Martínez, 1999).

El ciclo determina, diferencias significativas entre los escolares y no escolares en facetas vinculadas a ansiedad que podrían explicarse por el número de postulantes que se ha presentado anteriormente al examen sin éxito, entre los no escolares. Entre los colegios estatales y privados se evidencian diferencias en el factor Conciencia y en 
Personalidad, ansiedad estado-rasgo e ingreso a la universidad...

facetas diversas como sentido del deber, reflexión, complacencia, gregarismo y fantasía. En los colegios privados destaca fundamentalmente la faceta de calidez.

Se aprecian diferencias en las Escalas de Ansiedad únicamente en función de las variables sexo y ciclo. Las mujeres y los no escolares obtienen puntajes mayores tanto en la escala de Ansiedad- Rasgo como en la de Ansiedad- Estado. Las mujeres tienden a reportar con más facilidad las preocupaciones que las aquejan, y los no escolares pueden mostrar mayores índices de ansiedad en la medida que la mayoría postula por segunda o tercera vez al examen. Los alumnos del programa de Letras presentan puntajes significativamente más altos en la escala de Ansiedad- Estado.

La baja correlación entre la ansiedad autoreportada por los alumnos y reportada por el tutor podría deberse a la dificultad del tutor de reconocer diferencias entre la ansiedad como estado y como rasgo. De cualquier forma a pesar de tratarse de un grupo sometido a una presión intensa, ninguna de las escalas se eleva por encima de lo esperado, predominando una vivencia favorable que expresa la normalidad del grupo.

Al analizar la influencia de cada variable de manera independiente con el ingreso a la universidad se concluye que las variables que presentan una correlación positiva, significativa e independiente con el ingreso de los alumnos a la universidad son el factor Conciencia autoreportado por el alumno y reportado por el tutor, así como el $\mathrm{Cl}$, los cuales permiten determinar el ingreso de los alumnos con un $78 \%$ de acierto. En menor medida, la faceta sentido del deber reportada por el alumno. Asimismo se encuentra una asociación relativamente importante con el número de veces que el alumno postula, aunque ésta no supera la influencia que ejerce el CI.

El rendimiento intelectual medido a través de escalas de inteligencia muestra correlaciones significativas con la dimensión Apertura 
medida a través del NEO PI- $\mathrm{R}$. La relación con la inteligencia no sería directa; la asociación sería entre pensamiento divergente y habilidad creativa (Costa, McCrae, 1992). Apertura es el factor que más se asocia a inteligencia, habilidades cognitivas y creatividad (McCrae, 1985). Si bien no tiene una relación directa con la inteligencia, sí la tiene con pensamiento divergente y con aspectos motivacionales (Hogan, Johnson y Briggs, 1997; McCrae, 1987).

En términos generales, el dominio de tareas se encuentra vinculado a sentimientos de competencia y valía personal. En el ámbito universitario este dominio se expresa en términos del rendimiento efectivo. El éxito académico requiere una aproximación organizada, práctica y una atención cuidadosa para los procedimientos de análisis. En este sentido, resulta imprescindible el desarrollo de una autodisciplina personal y del sentido del deber. Los alumnos con un rendimiento inferior muestran más signos de inhibición, inseguridad y se describen como inquietos, irresponsables, con actitudes negativas hacia el centro de estudios, oposición a la autoridad y un ajuste emocional más pobre que el de los de mayor rendimiento.

El desenvolvimiento del alumno con más éxito coincide más con los valores y expectativas de su cultura, pero también puede generar mayor ansiedad: las metas altas pueden implicar que alguien no está conforme (Watson y Lindgren, 1992), lo que podría estar expresado con mayor claridad en las mujeres y estudiantes que ya postularon anteriormente sin éxito. Los estudios de Bergeman (1993) sugieren que el interés intelectual lleva a mayores niveles educativos, relacionando con intelecto, las habilidades cognitivas, inteligencia, perceptividad y conocimiento, habilidad de aprender y razonar, capacidad de entender y comprender. En nuestro grupo, coincide con los puntajes altos en aspiración de logro.

La exploración de nuevas alternativas de carrera más realistas o más acordes con los nuevos términos de definición personal se inicia con claridad en la etapa de preparación para el examen de ingreso a 
Personalidad, ansiedad estado-rasgo e ingreso a la universidad...

la universidad. Los retos académicos y sociales que se encuentran, llevan al crecimiento moral, intelectual y a una progresiva afirmación de la identidad sobre la base de nuevos valores y compromisos. La experiencia preuniversitaria provee básicamente una instrucción formal pero también genera adquisiciones que permiten cambios en el pensamiento, razonamiento, capacidad de enjuiciamiento moral e implicación con asuntos de la comunidad, a través del acceso a experiencias de un mundo en expansión, nuevas responsabilidades, nuevos contratos sociales, y nuevos compromisos (Watson y Lindgren, 1992). El programa de estudios ofrece nuevos discernimientos y maneras de pensar (Papalia, 1992) y los tutores proveen nuevos modelos. La vida preuniversitaria representa un período de descubrimiento intelectual y de enriquecimiento personal; en el grupo evaluado es evidente que el entorno del centro preuniversitario resulta favorable, propiciando el dominio de emociones positivas y ofreciendo un continente para las ansiedades propias de todo reto.

Finalmente, destacando los hallazgos más importantes del estudio, vemos que éste ofrece respaldo empírico para el Modelo de los Cinco Factores de Personalidad y su aplicabilidad en población preuniversitaria. Se confirma la presencia de cinco dimensiones básicas de personalidad, y se aporta la validez de constructo de dicho modelo. las diferencias en la conformación de ciertos riesgos específicos de la personalidad no lo afectan y parecen más bien evidenciar matices culturales.

Se ha podido determinar que en los jóvenes evaluados predominan rasgos y emociones positivas. A pesar de la situación de alta presión y exigencia, los rasgos de Extraversión y Conciencia son los más significativos mientras que los rasgos negativos de Neuroticismo y Ansiedad se presentan con mucho menos intensidad, justificándose el énfasis en un trabajo tutorial orientado hacia los aspectos saludables del grupo, y sólo en los casos específicos que lo soliciten hacia técnicas de manejo de la ansiedad. 
La variable intelectual resulta el factor más fuertemente asociado con el ingreso, hallazgo que respalda el esfuerzo del centro por evaluar esta variable cuando los alumnos recién ingresan. Entre los factores demográficos, resulta más probable el ingreso de alumnos que se han presentado más de una vez al examen. Este aspecto, asociado a la importancia del factor intelectual debe ser tomado en cuenta, justificándose una metodología diferenciada para el trabajo con grupos.

\section{Referencias}

Anchante, M. (1993). Ansiedad rasgo- estado en un grupo de alumnos de EEGGCC de la PUCP. Tesis de licenciatura en psicología no publicada. Pontificia Universidad Católica del Perú, Lima.

Avendaño, M. (1999). Dependencia al tabaco y ansiedad rasgo-estado en estudiantes de postgrado de una universidad privada de Lima. Tesis de licenciatura en psicología no publicada. Pontificia Universidad Católica del Perú, Lima.

Bergeman, C.S., Chipuer, H., Plomin, R., Pedersen, N., Mc Clearn, G.E., Nesselroad, J., Costa, P. y Mc Crae, R. (1993).Genetic and envoronmental effects on openness to experience, agreableness and conscientiousness. An adoption/twin study. Journal of Personality, 2, 23-37.

Cassaretto, M. (1999). Adaptación del inventario de Personalidad $N E O P I-R$. Tesis de licenciatura en psicología no publicada. Pontificia Universidad Católica del Perú, Lima.

Chau, C. (1998). Consumo de bebidas alcohólicas en estudiantes universitarios: motivaciones y estilos de afrontamiento. Tesis de Magister en psicología, no publicada. Pontificia Universidad Católica del Perú, Lima.

Claux, M. L . (1999). School performance in peruvian adolescents: A psychological perspective on person and context predictors. Tesis doctoral. Universidad de Nimega: Mediagroep KUN/AZN.

Costa, P .T. y Mc Crae, R. R. (1985). The NEO Personality Inventory Manual. Odessa, Florida: Psychological Assessment Resources. 
Personalidad, ansiedad estado-rasgo e ingreso a la universidad...

Costa, P. T. y McCrae, R. R. (1992). Revised NEO Personality Inventory (NEO PI-R) and NEO Five-Factor Inventory (NEOFFI). Professional Manual. Odessa, Florida: Psychological Assessment Resources.

Digman, J. M. y Takemoto- Chock, N .K. (1981). Factors in the natural language of personality: Reanalysis, comparison and interpretation of six major studies. Multivariate Behavioral Research, 16, 149-170.

Hernández, R., Fernández, C., y Baptista, P. (1991). Metodología de la investigación. México: McGraw-Hill.

Hogan, R., Johnson y J. Briggs, J. (1997). Handbook of personality psychology. San Diego: Academic Press.

John, O. P. (1990). The Big Five taxonomy: Dimensions of personality in natural languages and in questionnaires. En L. Pervin (Ed.), Handbook of personality: Theory and research (pp. 66-100). Nueva York: Guilford Press.

Lerner, S. (2000). Reflexiones en torno a la universidad. Lima: Pontificia Universidad Católica del Perú.

Martínez, P. (1999). Elección vocacional y personalidad a través del Psicodiagnóstico de Rorschach en universitarios. Tesis de Magister en psicología no publicada. Pontificia Universidad Católica del Perú, Lima.

McCrae, R. R. y John, O. P. (1992). An Introduction to the Five Factor Model and its applications. Journal of Personality, 60, 175-215.

Papalia, D. (1992). Desarrollo Humano. México: McGraw Hill. Rojas, R. (1997). Ansiedad, cólera y estilos de afrontamiento en portadores del VIH. Tesis de Magister no publicada, Pontificia Universidad Católica del Perú, Lima.

Spielberger, Ch. y Díaz-Guerrero, R. (1975). IDARE, Inventario de Ansiedad Rasgo-Estado. México: El Manual Moderno.

Ter Laak, J. F. (1996). Las Cinco Grandes Dimensiones de la personalidad. Revista de Psicología de la Pontificia Universidad Católica del Perú, 2, 129-181. 
Van Lieshout, C. y Haselager, G. (1991, junio). Personality Factors in CCQ-Descriptions. Ponencia presentada en el Simposio The Development of the Structure of Temperament and personality from Infancy to Childhood; NIAS, Holanda.

Watson, R. y Lindgren, H. (1992). Psicología del niño y del adolescente. México: Limusa. 